Janez Vodičar

University of Ljubljana, Slovenia

\title{
Rezension Józef Stala, Familienkatechese in Polen um die Jahrhundertwende. Probleme und Herausforderungen (Tarnow: Biblos 2008), 204 Seiten, ISBN 978-83-733267-4-3
}

Im Vorwort zu diesem Buch stellt der Bischof von Tarnow, Mons. Wiktor Skworc fest, dass es sich um einen wegweisenden Schritt für die Zukunft handelt. Die moderne Erziehung und Ausbildung sind dem religiösen Unterricht immer weniger zugeneigt. Ziele, die verschiedene Reformatoren der Schulsysteme in Europa zu verfolgen versuchen, sind größtenteils auf die reine Betonung einer Fachkompetenz in den Bereichen gerichtet, die gut vermarktet werden können. Das Können wird immer mehr nur zum Verkaufsartikel, der dem Menschen weder zum größeren Vergnügen und persönlicher Verwirklichung verhilft, noch ihm eine Antwort auf existenzielle Fragen bietet. Auch die Familie ist nicht mehr das, was sie in der guten katholischen Tradition war: ein Ort, wo man sich als Kind Gottes fühlte. Auf die Krise der modernen Familie darf es nicht nur soziologische oder ökonomische Antworten geben. Auch das Christentum ist gefordert, sich dieser Krise aktiv zu stellen. „Der religiöse, oder wie manche ihn auch nennen mögen, der sakrale Charakter der Familie geht nicht nur aus ihren gesellschaftlichen Funktionen und Aufgaben hervor, sondern vielmehr aus ihrer spezifischen Wesensart, eine grundlegende und elementare Verwirklichung von Liebe zu sein, ja sogar eine elementare Form der Existenz der Person als Person darzustellen“ (18). So ist es erforderlich, dass wir immer eine Möglichkeit haben, die Familie und die Erziehung unter einem wirklich christlich orientierten Gesichtspunkt zu betrachten. Das wäre nicht nur für die Katechese sondern für ganze Menschheit von Bedeutung, wenn wir wirklich auch weiterhin jeden Menschen als Person schätzen wollen.

Das vorliegende Buch mit dem Titel: FAMILIENKATECHESE IN POLEN UM DIE JAHRHUNDERTWENDE. PROBLEME UND 
HERAUSFORDERUNGEN wird wahrscheinlich kein Verkaufs-Bestseller werden. Es versucht aber für Christen den wichtigsten Weg aufzuzeigen, damit auch der moderne Mensch den Weg zu Christus und seine Erlösung dort finden wird, wo er den größeren Teil seines Lebens verbringen müsste - in der Familie. Die Familie ist heute nicht nur ein Problem für die Kirche - sie stellt auch einen Lösungsansatz dar. Wenn man die Katechese im breitesten Sinne als Erziehung zum Glauben versteht, sind wir uns, zusammen mit dem Autor des Buches, Dr. Jozef Stala, dessen bewusst, dass die Bewältigung der Aufgabe der religiösen Erziehung keinesfalls nur von der Schule erwartet werden kann. Der Autor ist seit einigen Jahren Professor an der Universität und konnte viele Erfahrungen in der Katechese sammeln. Er arbeitet in Tarnow und ist in Polen als Autor verschiedener Lehrbücher für Katechese bekannt. Sein Ausgangspunkt ist klar: Eltern, Eheleute, die ihre bei der Taufe erteilte Verantwortung für den Glauben ernst nehmen, müssen Katecheten sein und diese Aufgabe so verantwortungsbewusst erfüllen wie ihre Rolle als christlicher Ehemann und christliche Ehefrau.

Das Buch gliedert sich in fünf Kapitel. Nach der Einleitung werden die Probleme und Herausforderungen der Familie in Polen um die Jahrhundertwende vorgestellt. Der religiöse Charakter der Familie wird vom psychologischen, soziologischen und säkularen Gesichtspunkt aus betrachtet. Der Autor stellt fest, dass die Veränderungen in der religiösen Funktion der Familie die Erziehung vor neue Aufgaben stellen. Das zweite Kapitel bringt einen historischen Überblick über die katechetische Funktion der Familie. Auf Grund der Schwierigkeiten und der neuen Herausforderungen müsste die moderne Familie heute ihr Recht auf Katechese als Pflicht betrachten. „Als Kirche im Kleinen ist die Familie berufen, der Gesamtkirche dadurch ähnlich zu sein, dass sie der Welt ein Zeichen der Einheit gibt durch ihr lebendiges Zeugnis vom Königreich und Frieden Christi, den die gesamte Welt anstrebt" (53). So ist für die richtige Katechese die Familie ein wesentliches Subjekt, ohne das es kein wirkliches Wachstum im Glauben gibt. Das dritte Kapitel zeigt, wie diese 'Hauskirche' als katechetische Gemeinschaft funktionieren soll. Aus verschiedenen Kirchendokumenten und seinen Erfahrungen als Priester zeigt der Autor das Zusammenwirken von Familie und Pfarrgemeinde, wobei er die Pfarrgemeinde als eine Hilfestellung für die Familie sieht.

Das vierte und fünfte Kapitel zeigen auf, worin die Familienkatechese besteht oder bestehen soll. Zuerst ist sie eine Hoffnungsträgerin, wo alle, Familie, Pfarrei und Schule zusammen arbeiten müssten. Verschiedene Modelle und inhaltliche Aspekte zeigen uns, wie eine Familie als communio personarum leben könnte. 
Die christliche Familie, in der aus richtigem, lebendigem christlichem Glauben gelebt wird, ist auch eine Chance und Hoffnungsträgerin für die Menschheit. Der Autor schlägt dazu fünf konkrete Modelle vor: Das Modell der Intervention (die Zusammenarbeit der Eltern mit der institutionalisierten Katechese), das Modell der organisierten Familie (das normale alltägliche Familienleben wird im Licht der Offenbarung betrachtet), das ekklesiale Modell (es entwickelt sich dank Familie und Kirche), das Modell einer dynamischen Zusammenarbeit (es wird die Originalität jeder einzelnen Familie berücksichtigt), das Modell Matka katechetka (es geht um eine 'geistige Mutter', die die Funktion der Glaubensbildung übernimmt).

Das Schlusswort bringt eine Zusammenfassung: „Die Familienkatechese erlangt die ihr zueigene Spezifikation auf vielen Ebenen. Deshalb kann man vor allem davon sprechen, dass die Familienkatechese im Magisterium des Lebens und im Magisterium des Wortes geschieht. Denn die umfassendste Katechese, besonders für das Kind im Vorschulalter, ist ein von religiöser Atmosphäre durchdrungenes Familienleben, das durch das authentische Vorleben der Eltern geschaffen wird. Durch die Gesamtheit des Familienlebens, die sich dort abspielenden Ereignisse und vor allem durch das persönliche Zeugnis der Eltern und der weiteren Familienmitglieder wird Gott für das Kind erlebbar. Begleitende Worte werden dann lediglich zu einer notwendigen Hilfe den religiösen Gehalt tiefer zu enthüllen, den es mit umfasst. Und sie spielen dann eine wesentliche Rolle in der weiteren religiösen Entwicklung des Kindes: Denn sie zeigen die Schönheit des Lebens auf, obwohl immer wieder Schwierigkeiten auftreten, geben christliche Hoffnung angesichts von Leiden und von Problemen, lehren, wie man in frohen Augenblicken danken und bei Verfehlungen um Vergebung bitten kann"(155).

Trotz der großen Betonung der Rolle der Eltern, die sie bei der formellen religiösen Erziehung haben, gemeinsam mit den beruflichen Katecheten bei der Ausübung des Religionsunterrichtes in der Pfarrei wie auch in der Schule, stellt das Buch den Prozess einer dynamischen Erziehung für den Glauben mitten in der Familie selbst in den Vordergrund. Denn das Heranwachsen und damit das Suchen nach dem Glauben wird immer mehr vernachlässigt und manche Eheleute benötigen selbst einen katechetischen Prozess. Das gesellschaftliche Milieu unterstützt nicht selbstverständlich das religiöse Leben und bietet die Religionskenntnisse nicht so an wie einst. Für alle, die nicht aus Polen stammen, ist es überraschend, dass das Buch, das die Verhältnisse im heutigen noch immer katholischem Polen vorzustellen versucht, viele Anregungen auch für den viel früher säkularisierten Westen anbieten kann. Die angebotene Lösung dieses 
Werkes für eine immer weniger christlich geprägte Umgebung, was auch die kirchliche Dokumente betonen, ist eine Familienkatechese, die in gut präsentierten kirchlichen Richtlinien mehr und mehr zur Realität der Pastoral der ganzen Kirche wird. Die Familienkatechese ist in der christlichen Praxis des Lebens aus dem Glauben zwar nichts Neues, sie ist aber heute auf Grund der säkularen Umgebung und der veränderte Situation der Familie eine große Herausforderung. Der Autor verweist auf die Vor- und Nachteile der modernen Familie, die durch die moderne Gesellschaft bedingt sind, und auf die Notwendigkeit des katechetischen Zutritts, der primär auf die ganze Familie gerichtet ist. Dieser Zutritt muss die Natur der Familie berücksichtigen und so wird sie sich eben durch das gemeinsame Wachsen im Glauben in die Vollkommenheit des Lebens nach dem Glauben einschließen. Bei der Betonung der Katechese der Familie ist es dringend notwendig, eine lebhafte und verzweigte Familienkatechese zu entwickeln. Einzelne Familien dürfen sich nicht in sich einschließen und zum Selbstzweck werden. Das vorliegende Buch bietet uns eine ekklesiologische Lösung an: die Familie lebt den Glauben aus sich selbst auf ihre eigene Art und Weise, aber dabei ist sie in die lokale wie auch in eine breitere Kirchengemeinschaft eingebunden. Es handelt sich nicht um etwas Künstliches, sondern der ganze katechetische Prozess wird auf diese Weise sowohl anthropologisch wie auch theologisch in das Geheimnis des Menschen als Kind Gottes eingeschlossen: „Die Sendung der christlichen Familie strebt danach, das Evangelium weiterzugeben und es selbst auszustrahlen, so dass das Familienleben zu einer Schule der Nachfolge Christi wird. In der familiären Gemeinschaft, die von selbstloser Gesinnung und der Achtung vor der Würde jeder menschlichen Person inspiriert ist, vollzieht sich die Festigung einer authentischen und reifen Gemeinschaft von Personen (communio personarum)“"(136).

Zahlreiche Bemerkungen und Zitate aus der Fachliteratur können den interessierten Lesern dabei helfen, seinen Standpunkt zu diesem Thema zu vertiefen. Jedem, der die polnischen Verhältnisse nicht kennt, offenbart das vorliegende Werk die Bemühungen der Kirche in Polen um die Familienkatechese. Durch die vorgestellten Modelle kann jeder Anregungen und Lösungsmodelle für die Arbeit zu Hause finden. Das Buch enthält auch eine Zusammenfassung in verschiedenen Sprachen (Englisch, Französisch, Spanisch, Italienisch und Polnisch) so dass jeder etwas im Buch finden kann. Für Polen ist dieses Buch eine gute Einführung in die katechetische und pastorale Arbeit, die jedem Anregungen für seine konkrete Arbeit in einer Pfarrei bieten kann.

Es verbindet uns die Erkenntnis, dass eine richtig geformte Familienkatechese, die schon durchgeführt oder geplant ist, noch mehr die Familienwerte stärker 
betonen wird, die auch für die Gegenwart von großer Bedeutung sind. Bei einer richtig angelegten und durchgeführten Familienkatechese geht es um die Ergänzung und Bekräftigung der Fülle des Lebens der Familie als Grundzelle der religiösen Gemeinschaft oder wie der Autor sagt: der Hauskirche. Durch die Analyse und Vorstellung der passenden Methoden und Ziele einer solchen Katechese können wir mit dem Autor übereinstimmen, dass dies der Weg der Kirche ist, die auch die volle Offenbarung der Gottesliebe Christi immer mehr verwirklichen wird und die wir dem heute immer mehr entfremdeten Menschen anbieten können: „Je christlicher die Familie wird, desto menschlicher wird sie und desto menschlicher werden auch die Kultur und das Leben in der Gesellschaft" (129). 
